\title{
Applicability of the Bulge Equations in plane strain bulge experiments
}

\author{
J. Neggers $^{1, \text { a }}$, J.P.M. Hoefnagels ${ }^{1}$, and M.G.D. Geers ${ }^{1}$
}

Eindhoven University of Technology, Section Mechanics of Materials, P.O. Box 513, NL 5600 MB, The Netherlands

\section{Introduction}

The miniature plane strain bulge test is acknowledged as a powerful technique to study the mechanical properties of thin films [1,2]. A bulge experiment consists of measuring the deflection $\delta$ of a membrane under pressure $P$ (Fig. 1a). Full stress-strain curves can be calculated (Fig. 1b) using the Bulge Equations:

$$
\sigma=\frac{R P}{h}, \quad \varepsilon=\frac{R}{a} \arcsin \left(\frac{a}{R}\right)-1, \quad R=\frac{a^{2}+\delta^{2}}{2 \delta}
$$

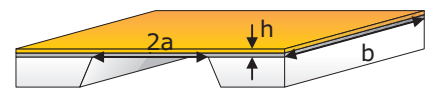

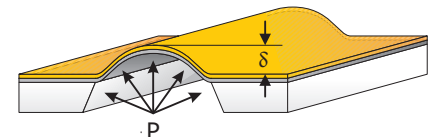

(a)

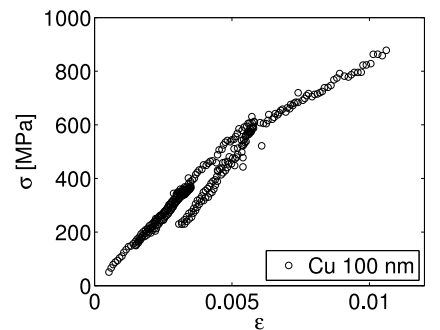

(b)

Fig. 1. a) Schematic of a plane strain bulge test experiment. b) Typical stress-strain curve from a bulge test experiment on $100 \mathrm{~nm}$ thick $\mathrm{Cu}$ film.

\section{Problem description and Project aim}

The bulge equations are based on the assumption that the membrane deflects to an ideal cylindrical shape. But for low stresses and/or thick films the membrane is better described with a Double Clamped Plate (DCP) solution.

The goal is then to assess when the bulge test experiment can accurately be described with an ideal cylindrical shape. Furthermore, the systematical error induced by use of the Bulge Equations is assessed quantitatively.

\footnotetext{
a e-mail: j.neggers@tue.nl
} 


\section{Methods}

Analytical analysis and 2D finite element analysis of the evolving bulge membrane profile, for different ratios of window width versus film thickness $\left(\frac{a}{h}\right)$.

\section{Results}

These simulations show that all profiles start from a DCP solution $\left[z=\frac{P}{E h^{3}}\left(-\frac{a^{4}}{2}+a^{2} x^{2}-\frac{1}{2} x^{4}\right)\right]$, and evolve towards a cylindrical profile (Fig. 2a), described by the Bulge Equations. Nevertheless, all profiles are cylindrical within a critical distance $a_{c}$ from the apex $(x=0)$.

A master plot was successfully constructed (Fig. 2b), which enables bulge test experimentalists to identify the applicability regime of the Bulge Equations for their bulge tests in terms of;

- Membrane dimensions

- Stress regime

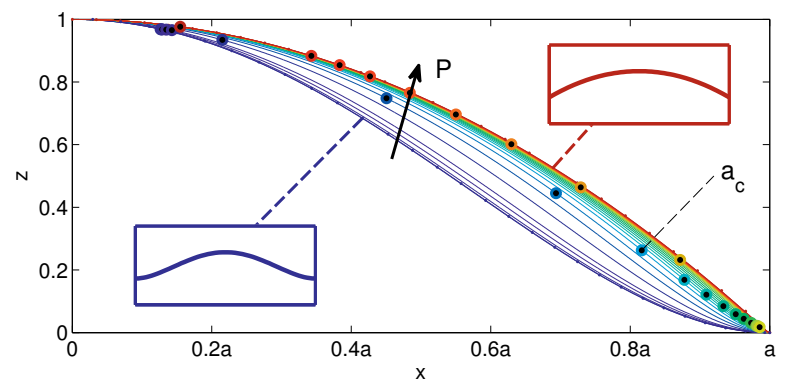

(a)

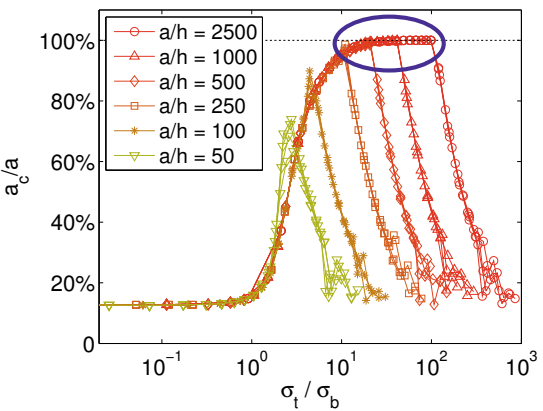

(b)

Fig. 2. a) Normalized bulge profiles for simulations of one side of a $1 \mu \mathrm{m}$ thick $(\mathrm{Cu})$ membrane, $a_{c}$ is indicated with dots. b) Master plot showing the relative circular part $\frac{a_{c}}{a}$ against the ratio of tangent $\sigma_{t}$ versus bending $\sigma_{b}$ stress; the Bulge Equations are only valid for the case that $\frac{a_{c}}{a}=1$, i.e. inside the ellips.

\section{Conclusions}

- Each experiment goes through an initial region where the Bulge Equations are invalid.

- The master curve shows directly in which regime the Bulge Equations are applicable.

- The Bulge Equations are never valid for films thicker than $2 \mu \mathrm{m}$ on standard bulge test setups [1,2].

- The systematical error induced by use of the Bulge Equations is identified.

\section{References}

1. J. J. Vlassak et al., Journal of Material Research, (1992)

2. Y. Xiang et al., Acta Materialia.,(2006) 